\title{
How advances in epidemiology are influencing older adult psychiatry
}

\author{
Natalie Shoham (1) \& Claudia Cooper \\ COMMENTARY ON... Epidemiology and mental illness in old age ${ }^{\dagger}$
}

\begin{abstract}
Natalie Shoham, MSc, is a Clinical Training Fellow in the Faculty of Brain Sciences at University College

London and a psychiatric registrar in Camden and Islington NHS

Foundation Trust, London. Claudia Cooper, PhD, is Professor of

Psychiatry of Older Age in the Faculty of Brain Sciences at University

College London and an honorary consultant psychiatrist in Camden and Islington NHS Foundation Trust, London, UK.

Correspondence Natalie Shoham. Email: natalie.shoham.16@ucl.ac.uk

First received 16 Sep 2019

Final revision 11 0ct 2019

Accepted 18 Oct 2019

Copyright and usage

(C) The Authors 2020
\end{abstract}

${ }^{\dagger}$ See this issue.

\section{SUMMARY}

Shortcomings of randomised controlled clinical trials include their high cost, which often precludes very long-term studies and very large populations, and ethical constraints of randomisation. Observational studies are a valuable alternative and we outline their use in epidemiological research to study very long-term effects of lifestyle and medication on dementia, to explore (using Mendelian randomisation) the association between Alzheimer's dementia and individual traits, and to evaluate populationwide health inequalities and lifespan changes in risk factors for psychiatric illness.

\section{DECLARATION OF INTEREST}

None.

\section{KEYWORDS}

Epidemiology; older adults; mental health.

Lilford \& Hughes (2019, this issue) provide a reminder that 'association does not equal causation' but that, given the practical barriers to conducting clinical trials, observational data remain key to understanding the determinants of illness. Such data can also provide insights into preventive and treatment strategies. Here we outline three areas in which observational studies are the primary source of evidence: assessing very long-term effects of interventions; evaluating effects of potentially harmful conditions where randomisation would be unethical; and evaluating population-wide and lifespan factors.

\section{Assessing very long-term effects of lifestyle changes on dementia}

One advantage of epidemiological studies is the potential for very long-term follow-up, relative to the short duration of most randomised controlled trials (RCTs). Even 3-year RCTs cost millions of pounds, so evaluating how lifestyle changes affect disease over decades needs a different approach. The recent positive public health message that up to one-third of dementia cases might be preventable if lifestyle risk factors were comprehensively addressed was based on epidemiological evidence (Livingston 2017). Epidemiological findings such as these have led to public health innovations - especially when there is a very low risk of potential harms yet considerable potential benefits to introducing new strategies or advice. They have also informed successful trials of intensive lifestyle and behaviour change interventions aimed at reducing the incidence of dementia (Ngandu 2015). Large-scale epidemiological research also provides a unique opportunity to evaluate geographical and temporal differences in disease incidence and prevalence. For example, that the prevalence of dementia in higher-income countries is falling could not be discovered through any other study design (Lilford 2019 , this issue).

\section{Ethical alternatives to RCTs: Mendelian randomisation}

As Lilford \& Hughes describe (Lilford 2019, this issue), there are many situations where it would be unethical to conduct an RCT. We cannot randomly select and expose a group of people to low educational attainment, or poor diabetic or antihypertensive care, to see whether they acquire higher risk of developing Alzheimer's disease. New Mendelian randomisation techniques may help. Mendelian randomisation has been used to explore the association between Alzheimer's dementia and a variety of traits. One example is predicted educational attainment, which was studied by Larsson et al (2017). In that study, single nucleotide polymorphism (SNP) variants identified through genetic studies as being associated with educational attainment were used as proxies for the exposure (predicted educational attainment). SNPs are clearly allocated to the individual before the outcome (Alzheimer's disease) has chance to develop. We can therefore be certain of their temporal relationship with the outcome. Additionally, SNPs are not influenced by other traits, and so confounding should not explain any association between them and the development of Alzheimer's disease. This means that by using SNPs as a tool to represent the exposure, the 
authors could be more confident in using observational data to attribute causality (Larsson 2017; Davies 2018).

Mendelian randomisation studies are not without limitations and, as with other study designs, need to be interpreted with caution. They are only suitable when an identifiable and well-understood genetic basis for the exposure variable exists. Another problem is that, since SNPs are only a proxy measure for the exposure and may individually confer very small effect on it, extremely large samples can be required to detect an association. Nevertheless, Mendelian randomisation studies are an exciting recent development in the field of epidemiology.

\section{Population-wide effects: health inequalities and changing risk factors over the lifespan}

Observational studies can observe effects of disease and take up of treatments over whole populations, rather than in the minority of people who volunteer to take part in RCTs (Cooper 2014). Even the most successful treatments work only if people take them, and in every area of medicine there are inequalities in access to good-quality care. For example, an observational study using data from general practice medical records reported that people from Black ethnic groups who are living in the UK were more likely to develop dementia than people from White ethnic groups, but they were less likely to receive a timely diagnosis (Pham 2018). Knowing this can guide public health strategies to reduce health inequalities. Of note, preliminary evidence that certain medications may influence dementia risk identified in observational studies is not always replicated in RCTs (Scharf 2007). This remains a challenge for observational studies.

Lilford \& Hughes' review describes functional illnesses in later life, highlighting that they can occur at different rates in older compared with younger adults. Epidemiological studies can explore not only differences in prevalence but also differences in risk factors at different points in the lifespan, as well as the underlying mechanisms. There is evidence that genetic susceptibility differs between late-onset and early-onset depression (Power 2017) and that late-onset depression may have a greater inflammatory component (Rozing 2019). It has been proposed that first-episode psychosis in old age can be a marker of underlying neurodegeneration (Van Assche 2017). Prevalence of risk factors may also differ by age group. For example, hearing loss appears to be associated with both dementia and schizophrenia, and sensory impairments occur more commonly in older people (Lilford 2019, this issue).

\section{Conclusions}

Lilford \& Hughes' helpful review highlights the importance of epidemiology to the mental healthcare of older adults. With the ever-increasing opportunities of big data for larger and more sophisticated observational studies, epidemiological data will continue to be a cornerstone of psychiatric research.

\section{Funding}

The UCL Division of Psychiatry is supported by the University College London National Institute for Health Research Biomedical Research Centre.

\section{References}

Cooper C, Ketley D, Livingston G (2014) Systematic review and meta-analysis to estimate potential recruitment to dementia intervention studies. International Journal of Geriatric Psychiatry, 29: 515-25.

Davies NM, Holmes MV, Smith GD (2018) Reading Mendelian randomisation studies: a guide, glossary, and checklist for clinicians. BMJ, 362 : k601.

Larsson SC, Traylor M, Malik R, et al (2017) Modifiable pathways in Alzheimer's disease: Mendelian randomisation analysis. BMJ, 359: 55375.

Lilford P, Hughes JC (2019) Epidemiology and mental illness in old age. BJPsych Advances, this issue.

Livingston G, Sommerlad A, Orgeta V, et al (2017) Dementia prevention, intervention, and care. Lancet, 390: 2673-4.

Ngandu T, Lehtisalo J, Solomon A, et al (2015) A 2 year multidomain intervention of diet, exercise, cognitive training, and vascular risk monitoring versus control to prevent cognitive decline in at-risk elderly people (FINGER): a randomised controlled trial. Lancet, 385: 2255-63.

Pham TM, Petersen I, Walters K, et al (2018) Trends in dementia diagnosis rates in UK ethnic groups: analysis of UK primary care data. Clinical Epidemiology, 10: 949-60.

Power RA, Tansey KE, Buttenschøn HN, et al (2017) Genome-wide association for major depression through age at onset stratification: Major Depressive Disorder Working Group of the Psychiatric Genomics Consortium. Biological Psychiatry, 81: 325-35.

Rozing M, Veerhuis R, Westendorp R, et al (2019) Inflammation in older subjects with early- and late-onset depression in the NESDO study: a cross-sectional and longitudinal case-only design. Psychoneuroendocrinology, 99: 20-7.

Scharf J, Daffner K (2007) NSAIDS in the prevention of dementia: a cache 22? Neurology, 69: 235-6.

Van Assche L, Morrens M, Luyten P, et al (2017) The neuropsychology and neurobiology of late-onset schizophrenia and very-late-onset schizophrenia-like psychosis: a critical review. Neuroscience \& Biobehavioral Reviews, 83: 604-21. 